\title{
Una Propuesta Práctica para la Inclusión de Estudiantes Ciegos en una Carrera de Ingeniería: Un Estudio de Caso
}

\author{
A Practical Proposal for the Inclusion of Blind Students in a Career \\ of Engineering: A Case Study
}

\author{
Katherine Palma-Picado * \\ Camila Delgado-Agüero \\ Tania Elena Moreira-Mora
}

Instituto Tecnológico de Costa Rica, Costa Rica

\begin{abstract}
El ingreso de un estudiante ciego a una ingeniería implicó nuevos desafíos para la educación universitaria en torno a la accesibilidad y la equiparación de oportunidades. Desde un enfoque inclusivo, a partir del estudio de un caso, se pretendió desarrollar una propuesta práctica para la inclusión de estudiantes con ceguera total en una carrera de ingeniería. Con una metodología cualitativa basada en las técnicas de juicio de expertos, la entrevista semi estructurada y el análisis documental se logró sistematizar y validar la información de diferentes fuentes, recolectada entre el 2012 y el 2018, durante las etapas de admisión, permanencia y graduación. Para la recolección de datos se utilizaron principalmente guías para orientar las entrevistas a jueces, docentes y equipos de apoyo. A partir de un análisis inductivo se generó una propuesta práctica derivada de las acciones ejecutadas durante este periodo, agrupadas en dos ejes conceptuales: gestión en accesibilidad y accesibilidad académica. La principal conclusión es la relevancia de la articulación de todas las acciones con las normativas vigentes para garantizar la accesibilidad y los apoyos educativos. Esta propuesta permitirá, a todas las personas involucradas en los distintos procesos universitarios, elaborar sus propias rutas de trabajo con estudiantes ciegos.
\end{abstract}

Descriptores: Admisión; Permanencia; Graduación; Discapacidad visual; Educación superior inclusiva.

\begin{abstract}
The attendance of a blind student to an engineering career means new challenges on the higher education around accessibility and equality in opportunities. From an inclusive approach, from a study case was trying to develop a practical proposal for the inclusion of students with full blindness in an engineering career. With a qualitative methodology based on criteria judges, the semi-structured interview and the documentary analysis, it was possible to systematize and validate the information from different sources. That was collected between 2012 and 2018 during the stages of admission, permanence and graduation. For data collection, questions were mainly used to guide the interviews with judges, teachers and support teams. A practical proposal was generated from the inductive analysis of the actions carried out during this period, that grouped into two conceptual axes: accessibility and academical accessibility. The main conclusion is relevance of coordination all actions with national regulations to ensure the educational support and the accessibility. This proposal will allow all the people involved in different university processes to create their own work routes with blind students.
\end{abstract}

Keywords: Admission; Permanence; Graduation; Visual disability; Inclusive education. 


\section{Introducción}

El Instituto Tecnológico de Costa Rica (ITCR) es una institución autónoma de educación superior universitaria pública de Costa Rica, que inició en 1973 y su oferta académica tiene un énfasis en las áreas de ingeniería. Su misión es contribuir con el desarrollo integral del país a través de la formación de profesionales, la investigación y la extensión en el campo científico, tecnológico y técnico.

En el 2012 cuando ingresó el primer estudiante con ceguera total a la carrera de Ingeniería en Computación, surgió la necesidad de garantizar la accesibilidad, con los ajustes respectivos en las etapas de: admisión, permanencia y graduación. Desde la intención de ingreso del estudiante fue necesario un trabajo interdisciplinario y articulado entre las diversas instancias responsables para responder a sus necesidades educativas.

Con el ingreso de este estudiante surgieron desafíos, tales como: ¿está preparada la universidad para atender las necesidades de personas con discapacidad visual? y ¿están debidamente capacitados docentes, autoridades, personal de apoyo y estudiantes de la comunidad para relacionarse de forma inclusiva y atender las demandas de esta población? Estos planteamientos también fueron analizados, entre otros, por García y otros (2016). Para responder a estas preguntas, en el presente artículo se plantea el objetivo de desarrollar una propuesta práctica para la inclusión de estudiantes con ceguera total, a partir de la información recolectada sobre las acciones de gestión de la accesibilidad y accesibilidad académica para personas con discapacidad visual, dado que existe escasa literatura sobre este tema en la educación superior y más aún en estudiantes de carreras de ingeniería.

Con un enfoque inclusivo esta propuesta permitirá a todas las personas involucradas en los distintos procesos universitarios, conocer un conjunto de acciones, respaldadas con un referente conceptual para elaborar sus propias rutas de trabajo con estudiantes ciegos. Como bien lo destacan Abejón y otros (2010), no se trata de acoger medidas excepcionales para las personas con necesidades educativas, sino en la adopción de un modelo de currículo que facilite el aprendizaje de todos los alumnos desde horizontes particulares y distintos, pero no por ellos menos legítimos.

\section{Revisión de la literatura}

La experiencia de inclusión se fundamentó en tres conceptos claves para la atención general del estudiantado con discapacidad, de acuerdo con las normativas nacionales en: discapacidad y accesibilidad para el caso particular de este estudio.

Según la Convención sobre los Derechos de las Personas con Discapacidad, artículo 1, las personas con discapacidad son aquellas que tengan deficiencias físicas, mentales, intelectuales o sensoriales a largo plazo que, al interactuar con diversas barreras, puedan impedir su participación plena y efectiva en la sociedad, en igualdad de condiciones con las demás personas (Asamblea Legislativa, 2008). En relación con las personas con discapacidad visual, la Comisión Interuniversitaria de Accesibilidad a la Educación Superior (CIAES, 2019) del Consejo Nacional de Rectores (CONARE) de Costa Rica, máxima autoridad del sistema público universitario en el país, la define como la dificultad que presentan algunas personas para participar en actividades de la vida cotidiana y que surge como consecuencia de la dificultad específica relacionada con una disminución o pérdida de las funciones visuales y las barreras presentes en el contexto en 
que se desenvuelve. Al respecto, se establecen dos condiciones: pérdida de visión funcional total (ceguera), o parcial (baja visión).

Otro concepto importante en este estudio es la accesibilidad, que para Boudeguer y otros (2010) se define como el conjunto de características que debe disponer un entorno urbano, edificación, producto, servicio o medio de comunicación para ser utilizado en condiciones de comodidad, seguridad, igualdad y autonomía por todas las personas. Para ello, en primer lugar, habría que hacer más accesible toda la comunidad educativa mediante la adaptación de sus entornos, programas y herramientas (Solla, 2013). Estos ajustes para la eliminación de barreras y obstáculos permitirán que todas las personas puedan, de manera independiente, participar en igualdad de condiciones (Bregaglio, 2015). Es una precondición para que las personas con discapacidad tengan una vida independiente a pesar de las posibles limitaciones funcionales que puedan tener.

En este marco se contemplan dos ejes conceptuales relevantes en esta investigación: la gestión en accesibilidad y la accesibilidad académica. El eje de gestión en accesibilidad, entendida como la adopción de todas las medidas pertinentes para asegurar que el acceso al entorno universitario sea en igualdad de condiciones. Esto implica la identificación de necesidades de los usuarios, la consecución de fondos, así como la planificación y articulación con diferentes instancias y actores responsables de la ejecución en las siguientes dimensiones: actitudinal, ambiente, información y comunicación, tecnologías de apoyo a la discapacidad, servicios y políticas. El objetivo de la gestión en accesibilidad es que el estudiantado pueda incluirse y participar de la vida universitaria de forma independiente y autónoma (Ley 8661, 2008, artículo 9). A continuación, se definen cada una de las dimensiones considerando la actualización de aspectos teóricos y técnicos del Consejo Nacional de Personas con Discapacidad (CONAPDIS) y la elaboración de Holst (2006).

Ambiente: es una condición que cumple un edificio, ambiente, objeto o instrumento para ser usado por todas las personas en sus actividades vitales con seguridad, comodidad y autonomía, sin necesidad de adaptaciones especializadas. El fin de la gestión en esta dimensión es que la persona con discapacidad pueda acceder al entorno sin encontrar barreras y obstáculos que limiten su participación social. Esto se contempla en el Índice de Gestión en Discapacidad y Accesibilidad (IGEDA, 2017) del CONAPDIS, que evalúa el nivel de cumplimiento de los derechos de las personas con discapacidad en Costa Rica, considerando el marco legal.

Actitudinal: incluye todas las acciones que promueven e incorporan principios y prácticas participativas para prevenir, eliminar la discriminación y posibilitar prácticas positivas hacia las personas con discapacidad, en el marco del modelo social de la discapacidad y los derechos humanos. Para Bausela (2009) y Conde (2014), esta área es la más difícil de trabajar porque hace referencia a las creencias, actitudes, prejuicios y comportamientos que tienen las personas, pero donde radica el cambio hacia un concepto de universidad que responde a valores y no sólo a la adquisición de saberes.

Información y comunicación: son todas las acciones que facilitan el intercambio de información accesible en el nivel interpersonal (LESCO, Sistema Braille, táctil, entre otros) e incluye la producción, el acceso y uso de mensajes y material accesible. Tal y como señalan Moriña y otros (2016), se busca que el estudiante con discapacidad pueda interactuar con las personas, documentos y material audiovisual de manera más inmediata y efectiva, al igual que el resto de sus compañeros. 
Tecnologías de apoyo a la discapacidad: contempla aquellos productos de apoyo, equipamiento y sistemas técnicos accesibles a todas las personas para aumentar, mantener o mejorar la independencia de las personas con discapacidad en todos los ámbitos de la vida diaria y la participación social. Corralesy otros (2016) y López (2019) coinciden en que su utilización para el proceso de aprendizaje es un apoyo que favorece la inclusión en el sistema universitario y es fundamental que respondan a las necesidades de las personas y los requerimientos del currículo; se eligen y configuran en relación con las tareas y competencias.

Servicios: se refieren a las acciones, recursos y medidas compensatorias para aumentar el grado de autonomía y garantizar oportunidades en condiciones de equiparación. Para Pérez (2019), los servicios institucionales son tan importantes como los ajustes al entorno físico porque son los que están en contacto directo con el estudiantado y están en la capacidad de valorar, asesorar y evaluar la gestión institucional orientada hacia el cumplimiento de derechos de las personas con discapacidad. Es más, sugiere la importancia de trascender la universidad y establecer alianzas estratégicas con otros servicios locales e instancias referentes en la temática.

Políticas: se refiere a todas aquellas orientaciones y lineamientos dirigidos a incorporar la normativa institucional, para el cumplimiento de la legislación nacional e internacional sobre discapacidad y accesibilidad. Esto lleva a la revisión, promoción, divulgación e incorporación de las políticas en la dinámica institucional. Como señala Devandas (2002), su existencia marca una diferencia en la orientación de las acciones y las estrategias para materializar lo que las universidades deben desarrollar transversalmente en todos sus procesos institucionales para transformarse en una universidad inclusiva. Desde esta conceptualización se logró sistematizar las acciones ejecutadas que facilitaron el ingreso y la permanencia del estudiante, al eliminar las barreras y brindar las oportunidades para garantizar una educación de calidad.

La segunda categoría es la accesibilidad académica y es concebida como aquel requisito que cumple una institución universitaria o educativa en general cuando se suprimen barreras que dificultan, obstaculizan o limitan la participación plena en las actividades académicas, así como el acceso a los servicios educativos y actividades cocurriculares, enmarcadas en el área de la atención a la salud, el deporte, el tiempo libre y el bienestar estudiantil (Mareño, 2005). Bajo esta línea, este concepto agrupa el abordaje pedagógico, los ajustes y apoyos para responder a las necesidades de la población con discapacidad (Fontana, 2016), asegurando el acceso, la permanencia y la graduación de todos sus estudiantes.

Desde esta perspectiva, en el estudio se asumió como el conjunto de requisitos relacionados con los apoyos educativos, metodología, materiales didácticos y evaluación, implementados en el ITCR para eliminar las barreras que dificultan la participación plena del estudiante ciego en las actividades académicas y en el uso de los bienes y servicios estudiantiles y educativos. Como lo señala Vindas (2013) estas barreras, por ejemplo, son las formas en que la falta de recursos o de competencias, el currículo o métodos de enseñanza inadecuados y las actitudes pueden limitar la presencia, participación y los resultados de estudiantes. Dentro de este eje conceptual se incluyen las siguientes dimensiones.

Apoyos educativos: son todas las acciones relacionadas con las adaptaciones del aula, materiales, asignación de recurso humano de apoyo y asesoría docente entre otros, ejecutadas por escuelas, docentes y entidades responsables para garantizar el cumplimiento de las condiciones de accesibilidad en el ámbito académico. Melero y otros (2019) coinciden con Pérez (2019) en señalar que cuando el profesorado tiene formación continua en atención a la diversidad, puede ajustar los materiales, desarrollar estrategias de apoyo educativo para responder a las 
necesidades del estudiantado con discapacidad e intervenir el proceso de aprendizaje con una actitud positiva para hacer los ajustes necesarios.

Metodología: son aquellas acciones ejecutadas para ajustar las estrategias metodológicas de un curso a las necesidades educativas de la población estudiantil con el fin de garantizar el acceso al currículum. Bajo esta línea, Paz (2018) señala que las instituciones de educación superior deben implementar nuevas modalidades de aprendizaje para construir mejores espacios educativos.

Materiales didácticos: ajuste de materiales físicos o digitales a las necesidades del estudiante para facilitar el aprendizaje. Como lo indican Cristian y otros (2017) en el proceso de construcción del conocimiento los materiales didácticos pueden guiar, evaluar, motivar, ejercitar o fomentar distintas habilidades. El acceso a la información se puede realizar a través de la adaptación de los materiales considerando los sentidos de la vista, del tacto y del oído (García, 2012).

Evaluación: incluye acciones que se efectúan para la comprobación, aplicación y acreditación de competencias profesionales. Al respecto Tejeda (2019) señala que la evaluación educativa en estudiantes en condición de discapacidad debe considerar las necesidades, problemas, negociaciones y toma de acuerdos consensuados. En suma, en este eje conceptual se analizan distintos elementos curriculares -objetivos, contenidos, estrategias didácticas o actividades, recursos didácticos, organización del tiempo y el espacio, criterios y procedimientos de evaluación- para dar respuesta a la diversidad de las condiciones particulares y posibilidades funcionales de cada estudiante (Abejón et al., 2010). A partir de este planteamiento conceptual se elabora una propuesta práctica con las acciones ejecutadas en el ITCR en las etapas de: admisión, permanencia y graduación. Esto permitirá que docentes, autoridades y colaboradores universitarios planifiquen acciones concretas para lograr la accesibilidad a cada una de las dimensiones, según las necesidades particulares del estudiantado ciego.

\section{Método}

En este estudio de caso se realiza una sistematización de las acciones ejecutadas, en consonancia con el referente conceptual de gestión en accesibilidad y accesibilidad académica. Como destaca Ary y otros (2010), el estudio de casos es un tipo de investigación etnográfica que se focaliza en un caso único o grupal, una organización o un programa. En este estudio se utilizaron diferentes técnicas para recolectar la información como el juicio de expertos, el análisis documental y la entrevista semiestructurada. En cada una de estas técnicas participaron expertos en discapacidad, personal docente y profesionales de apoyo de la institución, quienes colaboraron en las diferentes etapas del estudio; cuyo período inició con el ingreso del estudiante a la carrera de Ingeniería en Computación en el año 2012 y concluyó en el 2018 con su graduación.

La técnica de juicio de expertos se define como una opinión informada de personas con trayectoria en el tema, que son reconocidas por otros como expertos cualificados en este, y que pueden dar información, evidencia, juicios y valoraciones (Escobar y Cuervo, 2008). Específicamente, se contó con varios expertos de la universidad que integraban el Programa de Equiparación de Oportunidades, que vela para que se garantice el derecho al estudio y al trabajo en sus diferentes dimensiones; el Programa de Apoyo para la Admisión con Accesibilidad para Estudiantes con Necesidades Educativas y Discapacidad, que atiende las solicitudes de apoyos para la prueba de aptitud académica y el Programa de Servicios para Estudiantes con Discapacidad y Necesidades Educativas (PSED-NE), responsable de gestionar los apoyos y el acompañamiento psicoeducativo a lo largo de la carrera de los estudiantes hasta la graduación. 
La segunda fue el análisis documental, una forma de investigación técnica a partir del procesamiento analítico y sintético de la información, con fin de describir y representar los documentos de forma unificada y sistemática para facilitar su recuperación (Dulzaides y Molina, 2004). En este tratamiento documental se realizó la extracción y el análisis de la información registrada desde el 2012 hasta el 2018 en: el expediente de solicitud de adecuación para la prueba de aptitud académica del ITCR, la documentación del Comité de Examen de Admisión, del Programa de Equiparación de Oportunidades y del Programa de Servicios para Estudiantes con Discapacidad y Necesidades Educativas (PSED-NE) sobre los procesos de admisión, permanencia y graduación.

Con la técnica de la entrevista semiestructurada se profundizó en la información recopilada en la revisión documental a partir de las vivencias expresadas por los docentes y colaboradores que lo acompañaron durante la formación universitaria. Como lo explican Pievi y Bravin (2009) esta técnica se puede aplicar en una forma estructurada o semiestructurada. En ambas es necesaria una guía en la cual se apuntan las cuestiones a indagar que han de orientar la conversación. Para estos autores resulta una herramienta flexible, capaz de adaptarse a diferentes condiciones, situaciones, personas, permitiendo profundizar sobre las dimensiones de la investigación. Para ello se organizaron varias sesiones, de acuerdo con las personas responsables, en su momento, de cada uno de las etapas de admisión, permanencia y graduación.

- La primera entrevista se realizó el 17 de octubre del 2019 con los responsables de la construcción y aplicación de la prueba de admisión en el año 2011 y de los apoyos aprobados para el estudiante ciego. Las preguntas se orientaron hacia las adaptaciones de la prueba de admisión, su aplicación y el seguimiento en la etapa de inscripción.

- La segunda entrevista se realizó el 5 de diciembre del 2019 con docentes que impartieron diferentes cursos durante la carrera; con quienes se profundizó en la adaptación del material y pruebas, la estrategia didáctica, el uso de herramientas tecnológicas y la atención individual del estudiante.

Finalmente, la técnica utilizada para el análisis de la información fue la triangulación de datos. De acuerdo con García y otros (2016, p. 641), es "la más utilizada en la investigación social y su aplicación requiere de la obtención de información sobre el objeto de investigación, mediante diversas fuentes que permitan contrastar los datos recogidos". Considerando la relevancia de esta estrategia para el análisis, la verificación e interpretación de los datos, se desarrolló el siguiente procedimiento metodológico:

- Revisión y lectura de la documentación correspondiente a cada etapa: admisión, permanencia y graduación.

- Categorización y codificación de la información documental según las distintas dimensiones de accesibilidad académica: apoyos educativos, metodología, materiales didácticos y evaluación; mientras que en gestión en accesibilidad se organizaron en: ambiente, actitudinal, información y comunicación, tecnologías de apoyo a la discapacidad, servicios y políticas.

- Transcripción de las entrevistas realizadas y verificación de la fidelidad de las ideas expresadas por expertos y docentes.

- Categorización y codificación de la información de las entrevistas según las distintas dimensiones. 
- Revisión de todos los segmentos de textos codificados, tanto de las entrevistas como del análisis documental, para seleccionar los más relevantes por dimensiones.

- Integración de la información a partir de las dimensiones identificadas a priori, según los dos ejes conceptuales de la investigación: accesibilidad académica y gestión en accesibilidad. Esto permitió ordenar y comparar la información recolectada de las diferentes fuentes dentro de cada dimensión de los correspondientes ejes y en cada una de las tres etapas analizadas.

- Para garantizar la veracidad y transparencia de los hallazgos solo se integró la información con dos o más coincidencias o aproximaciones de las acciones ejecutadas en cada dimensión.

En suma, a partir de este análisis descriptivo inductivo de la información específica de las dimensiones hasta los ejes conceptuales; se logró generar una propuesta práctica para la inclusión de estudiantes con ceguera total en una carrera de ingeniería. Finalmente, se indica que todo el proceso de sistematización y análisis se realizó de manera manual, conforme con el protocolo establecido para la confidencialidad de la información de los participantes.

\section{Resultados}

\subsection{Etapa de admisión}

La admisión es la aceptación de estudiantes para realizar estudios en el Instituto Tecnológico de Costa Rica y está organizado en tres momentos: inscripción, realización de la prueba de aptitud académica (PAA) y la matrícula en la universidad. En este proceso colaboran un equipo interdisciplinario de las universidades estatales públicas de Costa Rica, organizados por la Comisión Interuniversitaria de Accesibilidad a la Educación Superior (CIAES) para evaluar todos los casos que requieren de alguna adecuación para realizar la PAA. Esta solicitud se puede realizar por dos vías: en línea o presencial, entregando la documentación respectiva en cualquier sede universitaria estatal del país.

Luego de la inscripción los estudiantes realizan la PAA del ITCR, la cual está compuesta por 50 ítems de matemática y 30 de verbal para medir diferentes categorías de razonamiento. La calificación obtenida en la prueba (60\%) se suma a la nota de la educación diversificada (40\%) para calcular la nota de admisión. Si los estudiantes obtienen una nota igual o superior a la nota de corte de cada carrera logran la condición de admitidos en la universidad. Esta modalidad se conoce con el nombre de admisión abierta (Oficina de Planificación Institucional, 2014). Para efectos de esta investigación las acciones en este proceso son sistematizadas por los ejes temáticos de gestión en accesibilidad y la accesibilidad académica (Cuadro 1).

En esta etapa fue fundamental tener como punto de partida el conocimiento detallado de la condición del estudiante y con el análisis previo de la información se logró definir las acciones de intervención de manera oportuna. Desde el momento en que se conoció la intención del estudiante de realizar el examen de admisión, la universidad asumió una responsabilidad institucional para garantizar la inclusión y la accesibilidad. Esto implicó una ruta de trabajo entre las instancias responsables para la capacitación del personal responsable, la adaptación de la prueba e inducción al estudiante para asegurar la aplicación del instrumento de admisión en condiciones de equidad. 


\section{Cuadro 1}

Propuesta práctica para la gestión en accesibilidad y la accesibilidad académica en la etapa de admisión Dimensiones gestión en accesibilidad

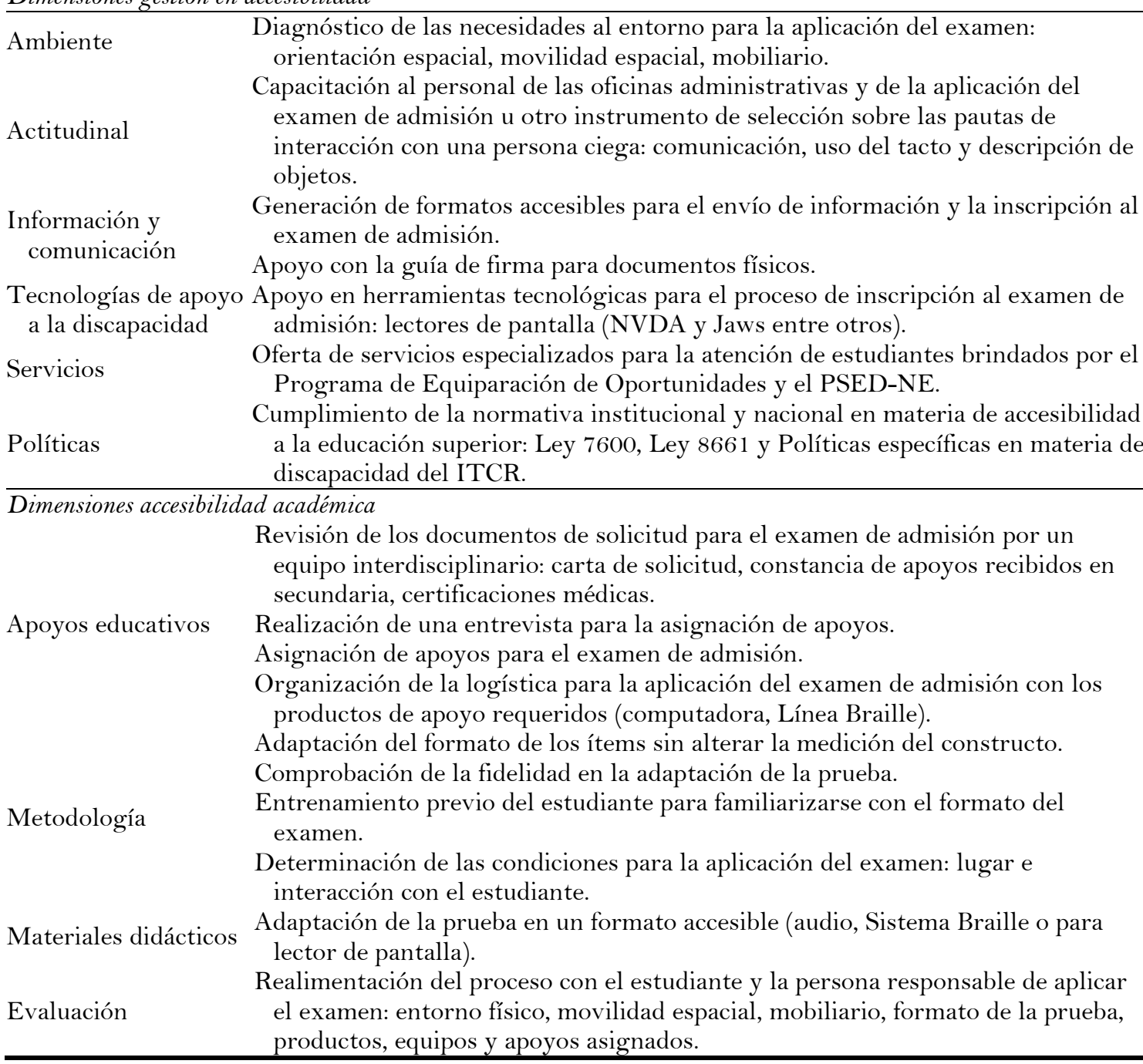

$\overline{\text { Nota. Expediente del estudiante y otros documentos del Comité Examen de Admisión, Programa de }}$ Equiparación de Oportunidades, del PSED-NE y aportes de expertos.

\subsection{Etapa de permanencia}

La permanencia es el periodo de formación universitaria del estudiantado que se inicia con la primera matrícula y culmina con la aprobación total del plan de estudio de la carrera. En el caso de esta investigación abarcó el periodo del 2012 al 2018 que, por la complejidad de la carrera de Ingeniería en Computación, implicó múltiples acciones en ambos ejes conceptuales, como se presenta en el Cuadro 2.

La gestión en accesibilidad durante la etapa de permanencia requirió un equilibrio entre las necesidades del estudiante y la capacidad de respuesta de la institución, conforme con la normativa y las políticas vigentes. Para ello fue necesario que los diferentes actores asumieran un rol activo a partir de la creación de redes de apoyo con los centros especializados para desarrollar las estrategias institucionales y pedagógicas. 


\section{Cuadro 2}

Propuesta práctica de la gestión en accesibilidad y la accesibilidad académica en la etapa de permanencia Dimensiones gestión en accesibilidad

individual, grupal, mobiliario y ajustes en infraestructura).

Apoyo con asistentes estudiantiles para la movilización dentro del campus universitario.

Entrenamiento en orientación y movilidad espacial dentro del campus y alrededores.

Ambiente Valoración del tipo de bastón a utilizar, según las características del terreno del campus.

Diagnóstico sobre la accesibilidad al espacio físico y validación de los ajustes con usuarios ciegos (perro guía y diferentes tipos de bastones).

Demarcación de rutas externas para el tránsito dentro del campus.

Colocación de losetas con relieve y otros materiales en zonas internas y externas para la movilización (tamaño, material, desgaste y color).

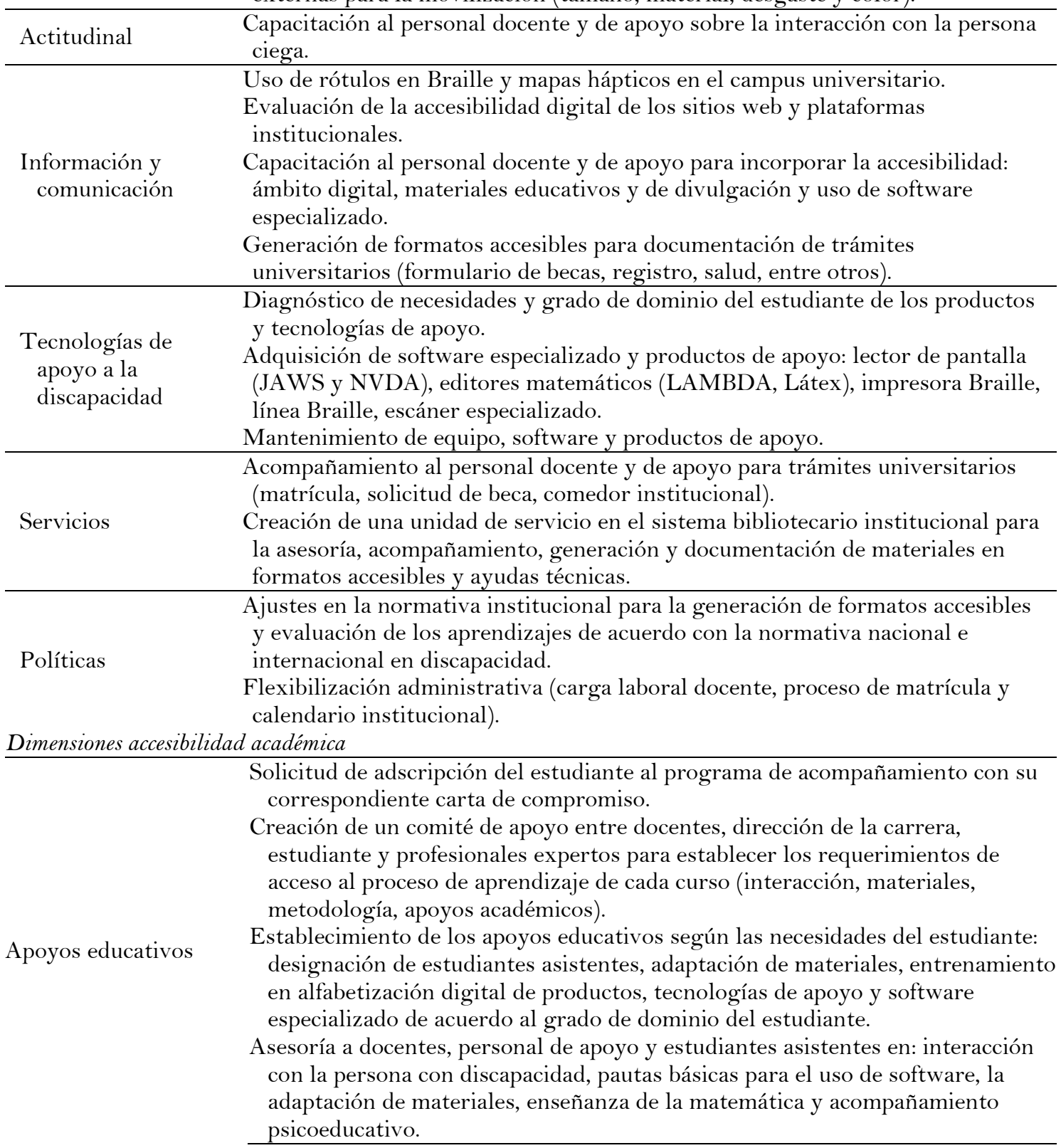




\begin{tabular}{c}
\hline Adaptación de materiales: digitalización de documentos, boletas de \\
recomendaciones de apoyos educativos, libros en formatos accesibles, entre \\
otros. \\
Asesoría psicoeducativa individual y grupal al estudiante ciego en el desempeño \\
académico y en las habilidades socioemocionales, durante cada período lectivo. \\
Evaluación de cada período lectivo para el mejoramiento de los diferentes \\
procesos y la toma de decisiones mediante un cuestionario enviado al \\
estudiante. \\
Realización de los ajustes metodológicos: verbalización de los elementos \\
gráficos y visuales en las explicaciones de clase, uso de herramientas \\
tecnológicas para la elaboración de materiales accesibles (JAWS, LAMBDA y \\
QuicTac), metodologías táctiles y kinestésicas para el aprendizaje de \\
contenidos. \\
Preparación de los materiales antes de las lecciones, o en el momento requerido, \\
para que el estudiante pueda participar de la dinámica de la clase. \\
Espacios de trabajo individual fuera de clase con el docente y estudiantes \\
asistentes, con el apoyo de medios digitales. \\
Uso y adaptación de materiales: sistema Braille, relieve, táctil, texto plano y \\
digital, imágenes, videos descriptivos, impresión en 3D y creación de códigos \\
de lectura de símbolos y fórmulas matemáticas, entre otros. \\
Comprobación de la accesibilidad de los materiales (windows, macos, online, \\
OCR y ABBYFineReader). \\
Validación de la adaptación de los materiales con el estudiante. \\
Generación de un repositorio digital y físico de los materiales adaptados. \\
Adaptación de todas las actividades de evaluación en formatos accesibles: \\
digital, sistema Braille u otros. \\
Flexibilidad en las condiciones para la aplicación y devolución de los resultados \\
de las distintas actividades: tiempo adicional, recinto aparte de ser necesario, \\
evaluación por tractos y calendario. \\
Devolución de resultados de trabajos y evaluaciones en formatos accesibles. \\
\hline Evaluación
\end{tabular}

Nota. Expediente del estudiante y otros documentos del Programa de Equiparación de Oportunidades, del PSED-NE y aportes de expertos.

Por otra parte, en la gestión académica fue fundamental la coordinación entre el equipo docente, estudiante y personal de apoyo para aprovechar los recursos institucionales y establecer acciones y recomendaciones de común acuerdo entre las partes. Esto implicó que todos los actores asumieran un papel activo y receptivo para aprender e investigar sobre la accesibilidad y la discapacidad.

También la planificación estratégica es relevante para la permanencia del estudiante al permitir su inclusión en el proceso de aprendizaje. Esta planificación consideró la diversidad en el aula, la dimensión actitudinal y el rol educativo del docente. Por ello la capacitación y asesoría debe ser una iniciativa integral e institucional que se enfoque en cuestiones técnicas y actitudinales, para lograr la concienciación de todas las partes involucradas y una experiencia exitosa y gratificante en estudiantes y docentes. No obstante, la disponibilidad de personal especializado en educación universitaria resulta clave en esta etapa; de lo contrario, es necesario buscar las colaboraciones con centros especializados externos.

\subsection{Etapa de graduación}

Esta última etapa corresponde a la gestión de trámites del título y la certificación de la conclusión de los estudios universitarios, la cual involucra un conjunto de acciones únicamente en gestión en accesibilidad, como se muestra en el Cuadro 3. 


\section{Cuadro 3}

Propuesta práctica de la gestión en accesibilidad en la etapa de graduación

\begin{tabular}{ll}
\hline Actitudinal & $\begin{array}{l}\text { Definición de pautas para la interacción con el estudiante durante el acto de } \\
\text { graduación. }\end{array}$ \\
\hline Ambiente & $\begin{array}{c}\text { Asesoría al estudiante y visita al espacio físico donde se realiza la graduación: } \\
\text { ingreso, ubicación y recorrido. }\end{array}$ \\
\hline Servicios & $\begin{array}{c}\text { Asesoría a personal encargado del acto de graduación: movilización dentro del } \\
\text { espacio físico, acompañamiento y ubicación, uso de plantilla de la guía de firma. } \\
\text { Comunicación a autoridades sobre ajustes al protocolo del acto de graduación. }\end{array}$ \\
\hline $\begin{array}{l}\text { Información y } \\
\text { comunicación }\end{array}$ & $\begin{array}{c}\text { Generación de la copia del título en formato accesible (Sistema Braille, audio, texto } \\
\text { plano). }\end{array}$ \\
\hline
\end{tabular}

Nota. Documentos del Programa de Equiparación de Oportunidades, Oficina de Admisión y Registro y aportes de expertos.

El proceso de acompañamiento concluye hasta el mismo día de la graduación, etapa que no puede descuidarse por ser la culminación de la formación universitaria. No hacerlo implicaría empañar todo el proceso que inició desde la aplicación del examen de admisión. Por ello es importante la asesoría a todas las instancias involucradas en las distintas actividades y los ajustes en los procesos que ya están estandarizados, sin alterar la formalidad del protocolo del acto de graduación.

\section{Discusión y conclusiones}

La inclusión y la accesibilidad parten del derecho a la educación como un derecho humano básico fundamental para la construcción de una sociedad más justa. Bajo esta premisa y ante el reto de tener un estudiante ciego por primera vez en la universidad, surgió esta investigación con la finalidad de generar una propuesta práctica inclusiva derivada del conjunto de acciones desarrolladas en la institución. Como bien lo destaca Bagnato (2017), la inclusión conlleva a la materialidad de los acontecimientos y a la eliminación de barreras físicas y comunicacionales, al amparo de la accesibilidad universal.

Una de las principales conclusiones es que la inclusión requiere voluntad política y de normativas que respalden a todos los actores y servicios; así como la coordinación interdepartamental para responder de manera eficiente a las necesidades del estudiante, docentes y personal de apoyo. Por ejemplo, en la etapa de admisión fue fundamental el contar con una normativa institucional y nacional para articular las acciones de diversas instancias responsables del examen de admisión, la asesoría especializada y los apoyos de herramientas tecnológicas, entre otros, que permitieron una aplicación exitosa de la prueba. De ahí la relevancia y necesidad de que las políticas institucionales y sus estrategias sean planeadas cuidadosa y detalladamente para asegurar que exista una correspondencia entre los objetivos y la ruta para lograrlos. A esta conclusión han llegado varios autores, quienes destacan que actualmente las personas con discapacidad pueden acceder a una formación profesional e integrarse al mundo laboral, en parte porque las leyes establecen que los centros educativos incorporen la temática de la discapacidad en todo el quehacer institucional (Ramírez, 2011). Además, las políticas universitarias vienen a enfatizar no sólo en la discapacidad como un tema aislado, sino también el de la inclusión, abriendo la puerta para el reconocimiento de los derechos de esta población y de otros grupos vulnerables (Carvajal, 2015). 
En correspondencia con lo anterior, si bien la universidad como institución debe ser activa en la atención de las necesidades, en este estudio la persona con discapacidad visual fue proactiva en el planteamiento y seguimiento a la resolución de los diferentes retos que plantea la vida universitaria. Esto porque la accesibilidad no es un proceso que se define en una vía, donde la institución determina lo que la persona requiere o responde según sus capacidades y limitaciones, sino que la clave de la inclusión y la equiparación es el resultado de la sinergia de la persona, sus condiciones, necesidades, fortalezas y oportunidades que la institución puede brindar. Al respecto, Gross (2016) señala que la información que se obtiene desde la perspectiva del estudiantado en relación con la accesibilidad al proceso educativo pone de manifiesto los alcances y limitaciones en el quehacer de la vida estudiantil y compromete a la universidad a mejorar ante los desafíos que enfrenta esta población. Así, plantea que las acciones se van construyendo paulatinamente en el contexto de la dinámica universitaria.

El ingreso y permanencia de un estudiante ciego en el sistema universitario, posiciona y fortalece los programas, servicios y a la institución como un sistema dinámico en el reto de la inclusión, convirtiéndose en un referente social en la generación de conocimiento, prácticas y modelaje. En este sentido, como señala Fajardo $(2017,195)$, una de las fortalezas de la educación superior en el nivel latinoamericano, es que "ha posibilitado espacios de participación, investigación, intercambio y divulgación de saberes y prácticas de inclusión de cada país, para continuar contribuyendo a la inclusión de las personas con discapacidad en la educación superior". Por su parte, Palmeros y Gairín (2016), rescatan que las experiencias e iniciativas inclusivas que se están sistematizando en las universidades, promueven la inclusión no sólo a lo interno, sino que también con la sociedad y el contexto. En el caso de la universidad, se fortaleció las competencias de los programas existentes y se creó uno adicional para reforzar las acciones en la etapa de admisión, así como la creación de una unidad de atención para personas con discapacidad en la biblioteca y otros proyectos relacionados con investigación.

Aunado a lo anterior, los profesionales fueron desarrollando experticia en la orientación y movilidad espacial, el uso de tecnologías de información y comunicación, ajustes en los apoyos educativos, adecuaciones en el ambiente físico, asesoría especializada al cuerpo docente, uso del braille y digitalización de materiales, entre otros. Al respecto Zárate y otros (2017), también reconocen la importancia de profesionales que articulen la participación de la comunidad educativa que interviene en la realidad social del estudiantado con discapacidad: personal docente y administrativo, y sus pares sin discapacidad.

El egreso y graduación también planteó la oportunidad y el reto de romper los prejuicios que existen en el nivel social para el ejercicio profesional de población con discapacidad. En este sentido, al ser la primera persona graduada en el país, específicamente de una ingeniería de una universidad pública, posibilitó su colocación laboral y la resignificación de la inclusión educativa en la enseñanza superior; como lo expresa Bagnato, (2017) esta representa el nivel más alto en la cadena educativa formal.

Los retos derivados de esta experiencia están relacionados en primer lugar con la capacitación docente para apoyar a esta población. La misma debe estar fundamentada en el principio de acceso con equidad e igualdad en educación y ser una actividad institucional permanente, porque va más allá de adaptar un material; implica conocer cómo relacionarse con una persona ciega o con baja visión, cómo planear una clase, cómo explicar, cómo realizar actividades grupales, de manera que el estudiante pueda participar en todos los espacios con autonomía e independencia. Además, porque el carácter activo y dinámico en las comunidades universitarias incluyen visitantes, invitados y nuevos estudiantes con esta condición. 
Una de las limitaciones encontradas en esta investigación fue la falta de sistematización del trabajo docente sobre la planificación de los cursos, la adaptación y el respaldo de los materiales utilizados; lo que implicó una reconstrucción a partir de las entrevistas hechas a docentes y expertos y las evidencias documentales encontradas en el expediente del estudiante. Por otra parte, debido a la prioridad de dar respuesta oportuna al proceso educativo, las personas responsables de cada programa no disponían de tiempo para organizar la información en sus bitácoras de trabajo; esto demandó más tiempo de las investigadoras en la revisión y organización de la información, especialmente en la etapa de permanencia.

Por último y desde el modelo social de la discapacidad, el hecho de responder a las necesidades del estudiante en las etapas de admisión, permanencia y graduación, condujo a que la universidad transversalice en su cotidianidad nuevos y diferentes aprendizajes, factores culturales, lengua, entre otros y los incorpore como parte de la vida universitaria (UNESCO, 2003). Tal y como se plantea a lo largo del artículo, sistematizar la experiencia y plasmarla en una guía que oriente la gestión en accesibilidad y la accesibilidad académica, permitirá contribuir a la producción de conocimientos y prácticas educativas inclusivas en otros contextos universitarios.

\section{Referencias}

Abejón, P., Martínez, M. y Terrón, M. (2010). Propuestas de acción para la integración de universitarios con discapacidad visual y auditiva ante el reto de Bolonia. Revista de Docencia Universitaria, 8(2),175196. https://doi.org/10.4995/redu.2010.6202

Ary, D, Cheser, L. y Sorensen, C. (2010). Introduction to research in education. Cengage Learning.

Bagnato, M. (2017). La inclusión educativa en la enseñanza superior: Retos y demandas. Educar en Revista, 3, 15-26. 196. https://doi.org/10.1590/0104-4060.51050.

Bausela, E. (2009). Actitudes hacia la discapacidad: estudio de algunas propiedades psicométricas en una muestra de universitarios mexicanos. Revista Iberoamericana de Educación, 49, 1-10.

Boudeguer, A., Prett, P. y Squella, P. (2010). Manual de accesibilidad universal: Ciudades y espacios para todos. Corporación Ciudad Accesible.

Bregaglio, R. (2015). El principio de no discriminación por motivo de discapacidad. En E. Salmón y R. Bregaglio (Eds.), Nueve conceptos claves para entender la convención sobre los derechos de las personas con discapacidad (pp. 57-89). Pontificia Universidad Católica.

Carvajal, M. (2015.) Política de discapacidad e inclusión de la Universidad del Valle: Un proceso participativo. Sociedad y Economía, 29, 575-20.

CIAES. (2019). Manual de procedimientos para la revisión conjunta. CONARE.

Conde, F. (2014). Desigualdad, discriminación y pedagogía de la igualdad. Revista Electrónica Actualidades Investigativas en Educación, 14(1), 1-20.

Consejo Nacional de Personas con Discapacidad. (2017). Informe de resultados índice de gestión en discapacidad y accesibilidad. IGEDA.

Corrales, A., Soto, V. y Villafañe, G. (2016). Barreras de aprendizaje para estudiantes con discapacidad en una universidad chilena. Demandas estudiantiles, desafíos institucionales. Revista Electrónica Actualidades Investigativas en Educación, 16(3), 1-29. https://doi.org/10.15517/aie.v 16i3.25957

Cristian, M., Vanina, G., Dupuy, R. y Mailén, D. (2017). Materiales didácticos inclusivos. Una mirada desde el diseño. Ponencia presentada en I Congreso Internacional de Enseñanza y Producción de las Artes en América Latina (CIEPAAL), Facultad de Bellas Artes, UNLP, Argentina. 
Devandas, C. (2002). Las personas con discapacidad en la educación superior: Una propuesta para la diversidady la igualdad. Fundación Justicia y Género.

Dulzaides, M. E. y Molina, A.M. (2004). Análisis documental y de información: Dos componentes de un mismo proceso. ACIMED, 12(2), 1-4.

Escobar-Pérez, J. y Cuervo-Martínez, A. (2008). Validez de contenido y juicio de expertos: Una aproximación a su utilización. Avances en Medición, 6, 27-36.

Fajardo, M. S. (2017). La educación superior inclusiva en algunos países de Latinoamérica: Avances, obstáculos y retos. Revista Latinoamericana de Educación Inclusiva, 11(1), 171-197. https://doi.org/10.4067/So7 18-73782017000100011

Fontana, A. y Rodríguez, R. (2018). Guía de apoyo para el personal académico de la Universidad Nacional que atiende estudiantes con deficiencia visual (ceguera o baja visión). Universidad Nacional.

Fontana, H. y Vargas, M.C. (2016). Las formas de apoyo educativo al estudiantado con discapacidad o con necesidades educativas en la Universidad Nacional de Costa Rica y sus implicaciones en su formación profesional. Revista Latinoamericana de Derechos Humanos, 27(2), 135-157. https://doi.org/10.15359/rldh.27-2.6

García, C. E. (2012). Guía de atención educativa para estudiantes con discapacidad visual. Instituto de Educación de Aguascalientes.

García, M., Maya, G., Pernas, I., Bert, J. y Juárez, V. (2016). La tutoría con enfoque inclusivo desde la universidad para estudiantes con discapacidad visual. Revista Cubana de Educación Superior, 3, 148160.

García, T., García, L., González, R., Carvalho, J. y Catarreira, S. (2016). Revisión metodológica de la triangulación como estrategia de investigación. Investigación cualitativa en Ciencias Sociales, 3, 639648.

Gross, M. (2016). Accesibilidad al proceso educativo en el entorno universitario. Actualidades Investigativas en Educación, 16, 1-17.

Holst, B. (2006). Estudio bibliográfico: Ayudas técnicas, tecnologías de apoyo y accesibilidad. Universidad Nacional

López, A. (2019). Accesibilidad académica en los ambientes virtuales. De las experiencias destacables a las políticas institucionales (Trabajo Final de Máster). Universidad Nacional de Mar del Plata, Argentina.

Mareño, M. (2005, junio). Accesibilidad académica en las instituciones de educación superior. Ponencia presentada en Jornadas de Tecnología y Discapacidad. Facultad de Ciencias Exactas Físicas y Naturales.

Melero, N., Moriña, A. y Perera, V. (2019). Acciones del profesorado para una práctica inclusiva en la universidad. Revista Brasileira de Educação, 24, 1-19.

Moriña, A., Cortés, M. D. y Molina, V. (2015). Educación inclusiva en la enseñanza superior: Soñando al profesorado ideal. Revista Latinoamericana de Educación Inclusiva, 9(2), 161-175.

Oficina de Planificación Institucional. (2014). Glosario institucional. Instituto Tecnológico de Costa Rica.

Palmeros, G. y Gairín, J. (2016). La atención a las personas con discapacidad en las universidades mexicanas y españolas, desde la revisión de las políticas educativas. Educación, 49, 83-102.

Paz, E. (2018). Competencias del profesorado universitario para la atención a la diversidad en la educación superior. Revista Latinoamericana de Educación Inclusiva, 12(2), 115-131.

Pérez, J. (2019). Tres modelos para ampliar las oportunidades educativas de personas con discapacidad. Discapacidad, inclusión social y educación. En J. Pérez y A. López, Discapacidad, inclusión social y educación (pp. 57-98). Instituto de Investigaciones sobre la Universidad y la Educación. 
Pievi, N. y Bravin, C. (2009). Documento metodológico orientador para la investigación educativa. Ministerio de Educación.

Ramírez, M. (2011). Las dimensiones de accesibilidad en la Universidad de Costa Rica Sede Rodrigo Facio. Un acercamiento desde las perspectivas de discapacidad y género. Reflexiones, 90(2), 71-88.

Reynaga-Peña, C. y Fernández-Cárdenas, J. (2019). La educación científica de alumnos con discapacidad visual: un análisis en el contexto mexicano. Revista Electrónica de Educación Sinéctica, 53, 1-17.

Solla, C. (2013). Guía de buenas prácticas en educación inclusiva. Save the Children.

Tejeda, P. (2019). La evaluación educativa en estudiantes en situación de discapacidad en la universidad: Desafíos y propuestas. Estudios Pedagógicos, 2, 169-178.

UNESCO. (2003). Superar la exclusión mediante planteamientos integradores en la educación: Un desafío y una visión, documento conceptual. UNESCO.

Vindas, O. (2013). Estrategias de atención de las necesidades educativas en la oferta de educación secundaria. Ministerio de Educación Pública.

Zárate, R., Díaz, S. y Ortiz, L. (2017). Educación superior inclusiva: Un reto para las prácticas pedagógicas. Revista Electrónica Educare, 21, 1-24. https://doi.org/10.15359/ree.21-3.15

\section{Breve CV de las autoras}

\section{Katherine Palma-Picado}

Profesora adjunta del Instituto Tecnológico de Costa Rica (ITCR) con una Maestría en Educación de la Universidad Framingham State College. Actualmente labora en el Departamento de Orientación y Psicología del ITCR, encargada del Programa de Servicios para Estudiantes con Discapacidad y Necesidades Educativas. Fue Directora de Proyectos de la Fundación DA Costa Rica (Déficit Atencional) y posee experiencia profesional privada en la atención de niños y jóvenes. Se ha desempeñado como profesional en psicología en el ámbito educativo, tanto en educación primaria, secundaria como universitaria y ha realizado labor docente en la Universidad Autónoma de Centro América. ORCID ID: https://orcid.org/oOoOoo03-3323-5714. Email: kpalma@itcr.ac.cr

\section{Camila Delgado-Agüero}

Profesora adjunta del Instituto Tecnológico de Costa Rica (ITCR) con una maestría en Psicología Clínica de la Universidad de Iberoamérica. Se ha desempeñado como profesional en psicología en el ámbito educativo, tanto en educación secundaria como universitaria. Ha realizado labor docente en la Universidad Latina de Costa Rica, la Universidad Nacional y la Universidad de Iberoamérica. Ha desarrollado investigación en los campos neuropsicológico y educativo. Actualmente trabaja en el Programa de Equiparación de Oportunidades del Instituto Tecnológico de Costa Rica como asesora psicoeducativa y tiene experiencia profesional privada con diferentes poblaciones. ORCID ID: https://orcid.org/OOOO-0002-3752-6327. Email: cdelgado@itcr.ac.cr

\section{Tania Elena Moreira-Mora}

Profesora asociada del Instituto Tecnológico de Costa Rica (ITCR) con un Doctorado en Educación de la Universidad Estatal a Distancia (UNED). Actualmente labora en el Departamento de Orientación y Psicología del ITCR, destacada en el Comité de Examen de 
Admisión y como docente en el Programa del Doctorado en Educación de la UNED. En el área de la evaluación ha fungido como asesora nacional de evaluación en el Ministerio de Educación Pública de Costa Rica, destacada en la evaluación de programas y proyectos educacionales. En investigación, las áreas de interés conciernen a la psicometría, el rendimiento académico, la evaluación educativa y la equidad. ORCID ID: https://orcid.org/0000-0002-8955-0804. Email: tmoreira@itcr.ac.cr 